\title{
Comportamiento de la arquitectura y flexibilidad muscular con el uso de kinesiotape en músculos gastrocnemios acortados en sujetos jóvenes: ensayo clínico randomizado \\ Behavior of muscle architecture and flexibility with the use of kinesiotape in shortened gastrocnemius muscles in young subjects: randomized clinical trial
}

\author{
Carolina Haydée Gajardo Contreras, Paula M acarena Caballero M oyano, Cristián Alejandro Caparrós \\ M anosalva, Jéssica Andrea EspinozaAraneda, Eduardo Jesús Soto Abarca \\ Universidad deTal ca (Chile)
}

\begin{abstract}
Resumen: 0 bjetivo: Anal izar los efectos del kinesiotape sobre la arquitectura y flexibilidad de los músculos gastrocnemios acortados en sujetos jóvenes durante tres días de aplicación. M étodo: Veinticinco jóvenes sedentarios con acortamiento del músculo gastrocnemio, fueron asignados al eatoriamente a un grupo experimental $(\mathrm{GE}=13$ ) intervenido con $\mathrm{KT}$ durante 72 horas y a un grupo control (GC=12). Se evaluó la flexibilidad del gastrocnemio y del sóleo. La arquitectura muscular, representada en la longitud del fascículo, grosor y ángulo de penación muscular, fue evaluada con un ultrasonógrafo en M odo $B$ en ambos grupos. Para determinar los efectos de KT, se aplicó una prueba t. Todos los análisis fueron con una significancia $p<0.05$. Resultados: ultrasonográficos mostraron un aumento significativo en la longitud del fascículo muscular ( $p=0.021)$, y una disminución en el grosor $(p=0.037)$ y ángulo de penación $(p=0.026)$ muscular post-intervención con KT en el GE. La flexibilidad de los múscul os gastrocnemio y sól eo aumento frente a la aplicación del KT pero sin cambios significativos. No se presentaron diferencias en el análisis entre el GE y GC. Conclusión: La aplicación de KT en sujetos con acortamiento de gastrocnemio, permite una mejor flexibilidad muscular al tercer día de uso, favorecido, posiblemente, por una modificación en la arquitectura muscular. Palabras claves: flexibilidad muscular, kinesiotape, ultrasonografía, gastrocnemios, músculo.
\end{abstract}

\begin{abstract}
To analyze the effects of kinesiotape on the architecture and flexibility of shortened gastrocnemius muscles in young subjects during three days of application. Method: Twenty-five sedentary youth with shortening of the gastrocnemius muscle were randomly assigned to an experimental group $(E G=13$ ) intervened with $\mathrm{KT}$ for 72 hours and to a control group ( $C G=12)$. The flexibility of gastrocnemius and sol eus was eval uated. The muscular architecture, represented in the length of the fascicle, thickness and angle of muscular pennation, was evaluated with a Mode B ultrasound in both groups. To determine the effects of KT, a t-test was applied. All analyzes were with a significance of $p<0.05$. Results: The ultrasonographic results showed a significant increase in the length of the muscular fascicle $(p=0.021)$, and a decrease in the thickness ( $p=0.037)$ and pennation angle $(p=0.026)$ post-intervention muscle with $K T$ in the $E G$. The flexibility of the gastrocnemius and soleus muscles increased compared to the application of $\mathrm{KT}$ but without significant changes. There were no differences in the analysis between the EG and CG. Conclusion: The application of KT in subjects with shortening of gastrocnemius, allows a better muscular flexibility on the third day of use, possibly favored by a modification in the muscular architecture. Keywords: muscle flexibility, kinesiotape, ultrasonography, gastrocnemius, muscle.
\end{abstract}

\section{Introducción}

El KT es un método terapéutico que ha mostrado mejorar la función muscular y articular en diferentes disfunciones motoras. El KT es un tape elástico que surge en el continente asiático en la década del 70 (Kase K, 2003). El KT presenta características físicas de grosor y peso similares a la piel y una capacidad de deformación de 130 a $140 \%$ de su longitud inicial (J., 2007). Se ha

Fecharecepción: 03-02-20. Fechadeaceptación: 08-11-20

Carolina Haydée Gajardo Contreras

cgajardo@ utalca.cl utilizado como una técnica facilitadora de la flexibilidad de músculos y tendones en desórdenes musculoesqueléticos de columna y extremidades, secuelas neurológicas entre otros (W itvrouw E, 2003).

La disminución de la flexibilidad muscular es un factor de riesgo importante para adquirir desórdenes musculoesqueléticos (W itvrouw E, 2003). Se traduce en una disminución de la longitud del músculo, lo cual se ha relacionado con menor rendimiento en activida des motoras (Arnold EM, 2013), limitando la movilidad y la calidad de vida. También se ha asociado con lesiones degenerativas y con menor capacidad de recuperación de una enfermedad o lesión, aumentando la probabili- 
dad de deterioro motor (Greve C, 2013). La flexibilidad de la musculatura del tríceps sural afecta la performance de miembrosinferiores en tareas habituales como la marcha y la carrera (Bohm, S, 2018, Arnold EM, 2013), la transferencia (Greve C, 2013), el balance (Gouveia ÉR, 2018), entre otras. También es causa de dolor en la región sural y limitación funcional que compromete a la rodilla y el tobillo (Siqueira CM, 2018).

La flexibilidad muscular del gastrocnemio, determinada por el rango de movimiento (ROM) de flexión del tobillo, ha mostrado relación con cambios en parámetros ultrasonográficos de la arquitectura muscular como el grosor, la longitud y el ángulo de penación del fascículo muscular. Un incremento en el ROM de flexión de tobillo se ha relacionado con una mayor longitud del fascículo muscular de gastrocnemios en baila rines (M oltubakk M M, 2018) y gimnastas (Donti 0 , 2019). Además, se ha reportado un efecto favorable en el RO M máximo de tobillo y el ángulo músculo-tendón del músculo gastrocnemio medial durante estiramientos estáticos al compararlos a estiramientos cíclicos en sujetos jóvenes (M aeda N, 2017).

Se ha reportado una mejora en la flexibilidad con el uso del KT de manera inmediata y a corto plazo (Cho HY, 2015). Efectos inmediatos favorables del uso del KT sobre la flexibilidad muscular han sido demostra dos, principalmente, en isquiotibiales (Espejo-Antúnez $L$, 2015) y gastrocnemios (Lumbroso D, 2014). Además, se ha señalado que el KT mejora la flexibilidad muscular alcanzando su mayor efecto al tercer día de uso (Farquharson C, 2015b; O zmenT, 2016).

Uno de los primeros estudios en mostrar datos ultrasonográficos del uso del KT fue LiuYH et al en el año 2007 que observaron que las imágenes de los músculos del extensor radial del carpo mostraron una disminución del grosor del fascículo duranteel estiramiento después de $24 \mathrm{~h}$ de uso. Por otro lado, Lin H-Y et al en el año 2015 observaron cambios en la excursión de la unión músculo-aponeurosis del gastrocnemio medial al utilizar el KT durante un salto vertical en sujetos sanos. Sin embargo, los cambios en la arquitectura muscular estudiados en el KT sobre la flexibilidad muscular no presentan consenso en sus efectos y han sido medidos principalmente post-aplicación. (Lin F-Y, 2015; LiuYH, 2007).

El 0 bjetivo de esta investigación es analizar los efec- tos del kinesiotape sobre la flexibilidad y arquitectura de los músculos gastrocnemios acortados en sujetos jóvenes sanos durante 3 días de aplicación.

\section{Método}

\section{Diseño de estudio}

Se realizó un ensayo clínico randomizado para determinar el efecto del KT aplicado durante 3 días en sujetos que presentaban acortamiento de gastrocnemio versus un grupo control. El estudio fue aprobado por el comité de ética científica de la U niversidad de Talca (Folio: 2017-14-CG) y conducido de acuerdo alos principios éticos para las investigaciones en seres humanos de la Declaración de Helsinki de la Asociación Médica Mundial (Versión Fortaleza, 2013).

\section{Participantes}

Los sujetos participaron voluntariamente y fueron reclutados a través de afiches y comunicados dispuestos en la universidad de Talca. Se utilizó una técnica de muestreo no probabilístico. Los sujetos reclutados completaron un formulario que considero los siguientes criterios de inclusión: hombres y mujeres, entre 20 y 30 años de edad, sedentarios (realizar menos de 150 sema nales de actividad física moderada 0 intensa) y la presencia de acortamiento del músculo gastrocnemio determinado como dorsiflexión de tobillo menor a $20^{\circ}$ con rodilla extendida la cual fue realizada por un profesional fisioterapeuta. Se excluyeron aquellos sujetos que presentaron diagnóstico de patologías ortopédicas, neuromusculares o neurológicas que afecten miembros inferiores en los últimos 6 meses, embarazo, alteraciones posturales y/ 0 malformaciones que limiten la realización de los test; consumir fármacos que alteren la extensibilidad de los tejidos (por ejemplo, relajantes musculares) y alergias atópicas o presencia de lesiones en la piel.

El tamaño de la muestra (G-Power 3.1.9.2, Universität Kiel, Alemania) se obtuvo utilizando los valores de flexibilidad con uso del KT durante $72 \mathrm{hrs}$, obtenidos en Caballero M oyano PM et al 2015, considerando una potenciadel $80 \%$ y un alfade 0,05 , obteniendo una muestra mínima de 14 personas por grupo. Finalmente, de los 28 sujetos seleccionados, se presentó una perdida en ambos grupos de 3 sujetos, por lo que el 
análisis considera en número final de 25 sujetos.

\section{Randomización}

Los 25 sujetos seleccionados (Tabla 1) fueron distribuidos aleatoriamente en un grupo experimental $(G E=13)$ con intervención de KT y un grupo control $(G C=12)$ sin intervención. La randomización fue rea lizada a través de una secuencia de números randomizados en un computador (http:// www. random.com).

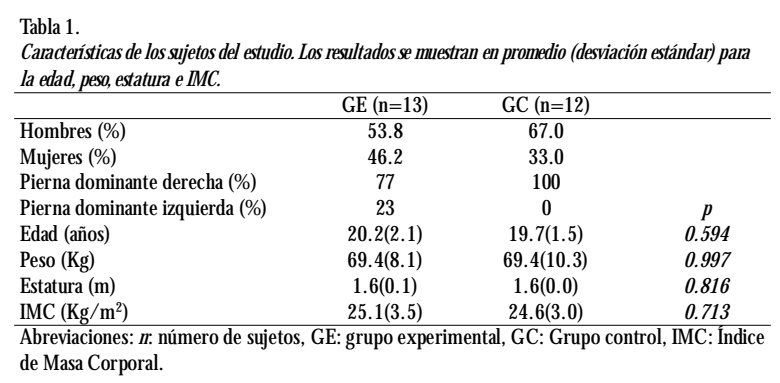

\section{Procedimiento}

El estudio se realizó en los Laboratorios de Musculoesquelético y Análisis de Movimiento Humano de la Escuela de Kinesiología de la U niversidad deTalca. Un integrante de los investigadores con más de 10 años de experiencia realizó la evaluación inicial y al tercer día a ambos grupos. Para determinar el efecto del KT sobre la flexibilidad muscular, se realizó una eval uación del rango de movimiento (ROM) de dorsiflexión de tobillo, con y sin flexión de rodilla. Posteriormente, se registró con ultrasonografía para obtener las variables de la arquitectura muscular del gastrocnemio.

\section{Pruebas de flexibilidad muscular}

Para determinar la flexibilidad del gastrocnemio se ubicó al sujeto en decúbito prono con la rodilla extendida y se registró el ROM de dorsiflexión (DF) con un goniómetro en grados. Adicionalmente, para discriminar el componente biarticular del tríceps sural (músculo sóleo), se midió el RO M de DF en la misma posición pero con rodilla en flexión de $90^{\circ}$. La validación de cada medición se realizó según los criterios descritos por Cejudo A et al 2015, que establece 4 condiciones: (a) incapacidad del evaluador de continuar el estiramiento por resistencia elevada; (b) disconfort relatado por el sujeto durante la prueba; (c) presencia de compensación y (d) aparición de dolor articular. Cada prueba fue realizada dos veces con un interval o de 30 segundos. Si los valores obtenidos presentaban una diferencia mayor al $5 \%$, se ejecutaba una tercera medición y se escogía el valor medio de los 2 valores más cercanos. Se seleccionó para la evaluación el lado con mayor acortamiento, en caso de ser iguales, se determinó el lado de dominancia a través de la prueba utilizada por W itvrouw et al el año 2003 (W itvrouw E, 2003). Todos los sujetos realizaron un calentamiento previo para evitar el riesgo de molestias durante la aplicación de las pruebas. En un cicloergómetro (Sportstart fitness c52u. Taiwan, China) sin carga, pedalearon por 5 minutos a 30 RPM. Luego se realizó ejercicios de estiramiento estático estandarizados en los grupos musculares implicados en el estudio.

\section{Prueba U Itrasonografía}

Se obtuvieron los parámetros de arquitectura muscular a través de la imagen del ultrasonógrafo (US) para el músculo gastrocnemio. Estos parámetros fueron la longitud del fascículo (LF), el grosor del músculo (GM) y el ángulo de penación (AP) del fascículo muscular. Se utilizó un ultrasonógrafo (M indray Z5. Mindray China) en M odo B y con un transductor configurado auna onda de frecuencia de $10 \mathrm{MHz}$. Se ubicó al sujeto en la posición prono para palpar el vientre muscular del gastrocnemio medial. El músculo estuvo relajado y el transductor se ubicó directamente sobre la piel del vientre muscular en su parte media determinada previamente y se utilizó gel conductor para obtener un mejor acople acústico del US. EI transductor fue posicionado perpendicular a la piel, paralelamente a los fascículos musculares del gastrocnemio en el tercio superior del segmento, se marcó la posición del transductor en la piel y se midió la distancia hacia el maléolo medial para reproducir la posición después de la intervención.

La LF yAP fueron determinados en la imagen registrada, donde se midió la distancia entre la aponeurosis superficial y profunda del epimisio muscular del gastrocnemio. La estimación de la longitud y ángulo de penación se realizó a través de la proyección del ángulo de las fibras hasta la aponeurosis con el procedimiento descrito por Farris DJ \& Lichtwark GA en el año 2016 con el software Ultratrack 4.1. El grosor se obtuvo directamente de la distancia de ambas aponeurosis en el vientre muscular. Todos los registros consideraron tres medidas de la imagen para cada evaluación. (Farris D), 2016). 


\section{Aplicación del KT}

Se utilizó un KT color cian, aplicado con una técnica muscular tipo «Y», con una base en calcáneo y 2 tiras sobre el trayecto del músculo gastrocnemio según las recomendaciones de Kase K et al del año 2003 (Kase K, 2003). Cada aplicación fue realizada por un experto certificado en terapia KT. Los participantes fueron instruidos sobre el adecuado uso del KT durante el tiempo de inter vención y de los posibles problemas que pudiesen ocurrir durante su uso. El KT fue aplicado por $72 \mathrm{~h}$, ya que es la duración que se ha observado el mayor efecto en la flexibilidad muscular y durabilidad de la aplicación (Caballero M oyano PM , 2015).

\section{Análisis Estadístico}

Las características de los sujetos y los resultados de las variables de ambos grupos fueron tabulados y presentados en media y desviación estándar. Se utilizó la prueba de Shapiro-W ilk para determinar si los datos provienen de una distribución normal y la prueba de Levene para observar la homogeneidad. Para determinar los efectos del VNM sobre las variables se utilizó la pruebat-student oW ilcoxon dependiendo del resultado de normalidad obtenido. Las comparaciones entre grupos fueron analizadas con pruebas para muestras independientes (GE vs GC), prueba t-student y de Mann-W hitney según correspondió. Adicionalmente, se calculó el tamaño del efecto para los cambios obtenidos con el VNM en el grupo intervenido, utilizando la pruebad deCohen (CohenJ, 1992) para la comparación con pruebas paramétricas y $r$ de Rosenthal (Rosenthal, 1991) para la comparación de pruebas no paramétricas. Se utilizó la clasificación de Hopkins (2002) como: 0.1, pequeño 0.2, mediano 0.6 , grande 1.2 y muy grande 2.0, para las pruebas paramétricas y para las pruebas no paramétricas se utilizó la clasificación de 0.10 pequeño, 0.30 mediano y 0.50 grande (Coolican H, 2009).

Todos los análisisfueron realizados con un nivel de confianza del $95 \%$ y un valor de $p<0.05$, utilizando el software SPSS (SPSS Inc., Chicago, IL, USA)

\section{Resultados}

De los 28 sujetos inicialmente seleccionados, 2 del GC y 1 del GE no cumplieron con retornar a la evaluación postintervención dentro de las 72 hr, por lo que fueron excluidos del análisis de datos, quedando una muestra de 25 sujetos distribuidos al eatoriamente en un GE y GC analizados. Las características de los sujetos del estudio (Tabla 1) presentaron que la edad, peso, estatura e IMC, no mostraron diferencias entre ambos grupos. La distribución por sexo, mostro un mayor porcentaje de hombresque mujeres en ambosgrupos, principalmente en el GC con un 67\%. Ambos grupos presentaron una mayor dominancia de la pierna derecha.

Los análisis de las variables entre los grupos e intragrupo, del ángulo de penación y grosor del fascículo fueron analizados con test paramétricos y las variables longitud del fascículo y flexibilidad con test noparamétricos. El estudio mostró cambios significativos en las variables de grosor, ángulo de penación y longitud del fascículo muscular y se observó un mayor rango de movimiento (ROM) en gastrocnemio y sóleo en el GE post aplicación del KT, no así en el GC. Sin embargo, estas diferencias no se presentaron al comparar ambosgrupos.
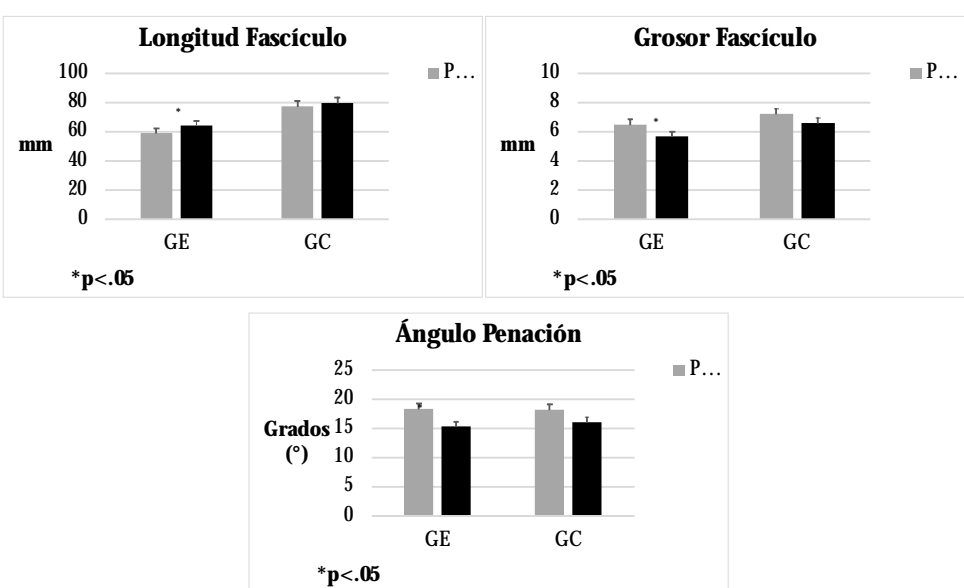

Figura 1. Las variables ultrasonográficas de longitud, ángulo de penación y grosor del fascículo muscular del gastrocnemio medial de ambos grupos, son presentadas antes y después de $72 \mathrm{~h}$ de aplicado el $\mathrm{KT}$.

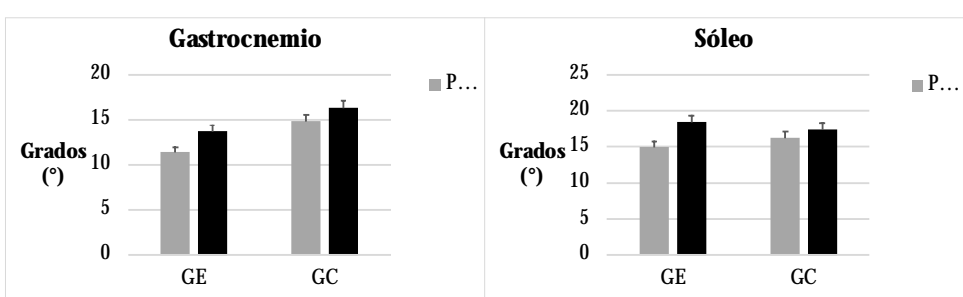

Figura 2. La flexibilidad muscular del gastrocnemio y sóleo de ambos grupos, representadas por el ROM, son presentadas antes y después de $72 \mathrm{~h}$. 
Resumen de las variables anal izadas con ultrasonografía y flexibilidad muscular durante la aplicación con KT.

\begin{tabular}{|c|c|c|c|c|c|c|c|c|c|c|}
\hline \multirow{2}{*}{ Parámetros } & \multicolumn{2}{|c|}{ GE $(n=13)$} & \multicolumn{2}{|c|}{$\mathrm{GC}(\mathrm{n}=12)$} & \multicolumn{2}{|c|}{$\begin{array}{c}\text { Intergrupo } \\
\text { Val or } p\end{array}$} & \multicolumn{2}{|c|}{$\begin{array}{l}\text { Intragrupo } \\
\text { Valor } p\end{array}$} & \multicolumn{2}{|c|}{$\begin{array}{c}\text { Tamaño del Efecto } \\
\text { Intragrupo GE }\end{array}$} \\
\hline & Pre & Post & Pre & Post & Pre & Post & $\mathrm{GE}$ & $\mathrm{GC}$ & dor & Clasificación \\
\hline \multicolumn{11}{|l|}{ Arquitectura M uscular } \\
\hline Longitud del Fascículo (mm) & $59.0(21.7)$ & $64.2(23.4)$ & $77.3(14.4)$ & $79.4(15.0)$ & .067 & .230 & .021 & .528 & -.640 & Grande \\
\hline Grosor del fascículo (mm) & $6.5(1.2)$ & $5.7(1.5)$ & $7.2(2.0)$ & $6.6(1.9)$ & .359 & .255 & .037 & .433 & .534 & Pequeño \\
\hline Angulo de penación $\left({ }^{\circ}\right)$ & $18.3(3.1)$ & $15.3(3.5)$ & $18.2(4.5)$ & $16.1(2.5)$ & .951 & .567 & .026 & .086 & .823 & Mediano \\
\hline \multicolumn{11}{|l|}{ Flexibilidad M uscular } \\
\hline$\overline{\text { Gastrocnemio }}\left({ }^{\circ}\right)$ & $11.4(1.5)$ & $13.7(3.3)$ & $14.8(3.8)$ & $13.6(2.7)$ & .083 & .965 & .091 & .400 & -.468 & Grande \\
\hline Sóleo $\left({ }^{\circ}\right)$ & $15.0(3.8)$ & $18.4(5.2)$ & $16.3(3.7)$ & $17.4(2.9)$ & .460 & .829 & .066 & .326 & -.509 & Grande \\
\hline
\end{tabular}

ratura, mostrando un aumento del ROM al $3^{\circ}$ día de aplicación post-inter vención, pero sin cambios significativos. Los posibles mecanismos que explicarían el aumento de la flexibilidad con el uso del KT han sido discutidos en los últimos años. El cambio en la flexibilidad puede ser consecuencia de la tensión continua que provocaría el KT sobre la piel, la cual afectaría

Los resultados ultrasonográficos (Figura 1 yTabla 2) mostraron un aumento significativo en la longitud del fascículo muscular ( $p=0.021$ ) con la aplicación del KT en el GE con un efecto del tamaño grande. El grosor muscular y el ángulo de penación del fascículo, mostra ron una disminución significativa post-intervención ( $p=0.037$ y $p=0.026)$ con un tamaño del efecto pequeño y mediano, respectivamente. Por otro lado, el ROM medido (Figura 2) de los músculos sóleo $(p=0.066)$ y gastrocnemio ( $p=0.091)$ mostraron un aumento de la flexibilidad de ambos músculos frente a la aplicación del $K T$, pero sin diferenciassignificativas post aplicación tanto en el GE como al comparar con el GC.

\section{Discusión}

El presente estudio investigó el efecto del KT en la arquitectura y flexibilidad muscular en el músculo gastrocnemio acortado. Los resultados mostraron un incremento en la flexibilidad muscular, longitud del fascículo y una disminución en el grosor y ángulo de penación del fascículo muscular, posterior a 3 días de aplicación del KT.

El aumento de la flexibilidad muscular con el uso del KT se ha reportado en musculatura de miembro inferior como isquiotibiales y gastrocnemio (Lumbroso D, 2014), en músculos acortados (Caballero M oyano PM, 2015; Espejo-Antúnez L, 2015), en condicionesmáscrónicas como artrosis de rodilla (Cho HY, 2015), entre otros. Además, se ha comparado con entrenamientos de estiramientos (Farquharson C, 2015a) y frente a la recuperación de ejercicio excéntrico (0 zmenT, 2016). A parentemente, la mayor ganancia de RO M con el KT ocurriría posterior a 2 días por sobre un efecto inmediato a su aplicación (Caballero Moyano PM, 2015; Farquharson C, 2015b).

Los resultados concuerdan con lo señalado en la lite- la continuidad de la fascia superficial (TurrinaA, 2013) y su relación con las fibras musculares insertas a la fascia que recubre al músculo y la septa intramuscular (Stecco C, 2007). Esta acción subcutánea, disminuirá la carga tensil sobre la fascia y favorecería el desplazamiento de los tejidos superficiales. Se requieren nuevos estudios que puedan analizar estos cambios mecánicos del tejido muscular y conectivo con el uso de KT.

Se han observado cambios en la arquitectura muscular del gastrocnemio en sujetos que trabajan de ma nera frecuente la flexibilidad muscular (Donti 0, 2019; M oltubakk M M , 2018) quienes presentaron un aumento en la longitud del fascículo muscular relacionado con un mayor ROM de flexión de tobillo. Por otro lado, otros estudios han reportado una disminución en el ángulo tendón-músculo, obtenido con US, cuando se intervinieron sujetos con ejercicios de estiramiento está tico (Maeda N, 2017). En este estudio, el uso de KT mostró un aumento en la longitud del fascículo y una disminución en el grosor y ángulo de penación.

Los cambios ocurridos en la arquitectura muscular hipotéticamente podrían estar relacionados con el aumento de la flexibilidad muscular, debido a una mayor longitud muscular que podría implicar un estado tensil menor, provocando una disminución en la distancia de sus fascias relacionadas, provocando por consiguiente un aumento de la longitud y un menor ángulo de penación del fascículo. El cambio de configuración podría tener explicación en loscambios de tensión sobre lafasciaplanteados con el uso de KT y sobre el estímulo táctil provocado por el KT sobre mecanorreceptores, provocando una modificación en la respuesta del músculo. Aún falta información que permita comprender estos meca nismos.

Los cambios en la arquitectura muscular se han señalado como un factor clave en las modificaciones de la flexibilidad muscular (Donti 0, 2019) y constituyen un 
factor relevante en deportistas como voleibolistas, sprinter, gimnastas, entre otros (AbeT, 2000; Lee SS, 2009). Existen pocos estudios que hayan analizado los cambios ultrasonográficos en el gastrocnemio con el uso de KT (Lin F-Y, 2015). Los resultados de este estudio mostraron un cambio en la arquitectura del fascículo muscular, lo cual podría orientar hacia el aporte tera péutico que podría tener este tipo de intervención.

Dentro de las limitaciones del estudio, se considera: el pequeño tamaño de la muestra, lo cual podría limitar los al cances de los resultados; el registro de la medición ultrasonográfica que fue realizado sólo en una posición del tobillo, lo que no permite analizar cambios de acuerdo a la longitud muscular; las diferencias basales de longitud muscular entre el GE y GC, que se generaron posiblemente debido a la randomización de los gruposy el no incluir un grupo con placebo o grupo sin acorta miento, que no permite determinar si el efecto es dependiente de la condición inicial del músculo. Por otro lado, es posible que los resultados sean explicados por otras variables no contempladas, sobre todo en las actividades que realizaron los sujetos en los tres días donde no se evaluó.

El combinar el KT con métodos de entrenamiento de flexibilidad permitiría comprender el aporte tera péutico de esta técnica asociada a intervenciones ya propuestas por la literatura, como por ejemplo técnicas de estiramiento, con efectos favorables cuando la muscula tura presenta una disminución de la flexibilidad. Los futuros estudios debieran considerar aspectos de técnicas de intervención concurrente, con el propósito de identificar sistemas eficientes en el tratamiento de este tipo dedisfunciones.

\section{Conclusión}

La aplicación de KT en sujetos con acortamiento de gastrocnemio, permite una mejor flexibilidad muscular al tercer día de uso, favorecido, posiblemente, por una modificación en la arquitectura muscular. El acortamiento de la musculatura de miembros inferiores compromete su flexibilidad aumentado el riesgo de lesiones músculo esqueléticas (Bahr $R$, 2003). La recuperación y mantención de la flexibilidad muscular del gastrocnemio es de gran interés para los terapeutas, por lo que ampliar las opciones de tratamiento o pre- vención a través del uso de KT permitiría mejorar la performance muscular y con ello disminuir su exposición a lesiones.

\section{Referencias}

AbeT, K. K., BrechueW F. . (2000). Fascicle length of leg muscles is greater in sprinters than distance runners. Med. Sci. Sports Exerc, 2000(32), 1125 1129. DO I: 10.1097/ 00005768-200006000-00014 Arnold EM , H. S., SethA, M illard M, Delp SL. . (2013). How muscle fiber lengthsand velocities affect muscle force generation as humans walk and run at different speeds. J Exp Biol., Jun 1, 2150-2160. D O I: 10.1242/ jeb. 075697

Bahr R, H. I. (2003). Risk factors for sports injuriesa methodological approach. Br J Sports Med, 37, 384 - 392. DO I: 10.1136/ bjsm.37.5.384

Bohm, S., Marzilger, R., Mersmann, F., Santuz, A., \& Arampatzis, A. (2018). O perating length and velocity of human vastus lateralis muscle during walking and running Scientific reports, 8(1), 5066. https:/ / doi.org/ 10.1038/ s41598-018-23376-5

Caballero Moyano PM, C. M. C., Rojas M atthei DA, CorreaBeltrán GX, Gajardo Contreras CH. (2015). Efecto del vendaje neuromuscular sobre el acortamiento de los músculos isquiotibiales. Fisioterapia $\mathrm{E}$ Pesquisa, 37, 105 - 111. DOI: 10.1016/ j.ft.2014.07.003

Cho HY, K. E., Kim J,YoonYW. . (2015). Kinesio taping improves pain, range of motion, and proprioception in older patients with knee osteoarthritis: a randomized controlled trial. Am J Phys M ed Rehabil, $M \operatorname{ar}(3), \quad 192-200 . \quad$ DOI: $10.1097 /$ PHM. 0000000000000148

Cohen, J. (1992). A power primer. Psychological Bulletin, 112(1), 155-159. doi:10.1037/ 00332909.112.1.155

Coolican, H. (2009). Research methods and statistics in psychology (5th ed.). Hodder Education Group.

Donti O, P. I., Terzis G, Bogdanis GC. (2019). Gastrocnemius Medialis Architectural Properties at Rest and During Stretching in FemaleAthletes with Different Flexibility Training Background. Sports (Basel), Feb(7), 2. DOI: 10.3390/ sports7020039 Espejo-Antúnez L, L.-M . P., Garrido-Ardila EM , Casti- 
Ilo-Lozano R, Domínguez-Vera P, Maya-Martín J, Albornoz-Cabello M. (2015). A comparison of acute effects between Kinesio tape and electrical muscle elongation in hamstring extensibility. J Back Musculoskelet Rehabil., 28(1), 93-100. DO I: 10.3233/ BMR-140496.

Farquharson C, G. M. (2015a). Temporal efficacy of kinesiology tape vs. Traditional stretching methods on hamstring extensibility. Int I Sports Phys Ther, $\mathrm{Feb}(1), 45$ - 51.

Farquharson C, G. M. (2015b). Temporal patern of kinesiology tape efficacy on hamstring extensibility. Int J Sports PhysTher, Dec(7), 984-991.

Farris DJ, L. G. (2016). UltraTrack: Software for semiautomated tracking of muscle fascicles in sequences of B-mode ultrasound images. Comput Methods Programs Biomed, May(128), 111 - 118. DOI: 10.1016/ j.cmpb.2016.02.016

GouveiaÉR, G. B., IhleA, Kliegel M, MarquesA, Freitas DL. (2018). Balance and mobility relationships in older adults: A representative population-based crosssectional study in Madeira, Portugal. Arch Gerontol Geriatr., Jan - Feb(80), 65 - 69. DOI: 10.1016/ j.archger. 2018.10.009

Greve C, Z. W., Hortobágyi T, Bongers RM. . (2013). Not all is lost: old adults retain flexibility in motor behaviour during sit-to-stand. PLOS O ne, 0 ct 25(e77760). DO I: 10.1371/ journal. pone. 0077760

Hopkins, W.G. (2002) A Scale of Magnitudes for Effect Statistics: A New View of Statistics. http:/ / www. sportsci.org/ resource/ stats/ effectmag. html

J., S. (2007). Manual deTaping neuromuscular. Madrid: Aneid-Press.

Kase K, W. J., Kase T (2003). Clinical Therapeutic Applications of Kinesio Taping M ethod. Tokio: Ken Ikai Co Ltd.

Lee SS, P. S. (2009). Built for speed: M usculoskeletal structure and sprinting ability. J. Exp. Biol., 2009, 3700 - 3707. DO I: 10.1242/ jeb. 031096

Lin F-Y, W. S.-F., Chen S-H, Chai H-M. . (2015). Changes in architecture of muscle-tendon unit and performance of gastrocnemius muscle gastrocnemius using Kinesio taping. Physiotherapy, 101, e878 - e879. D Ol:https://doi.org/10.1016/ j.physio.2015.03.1709

LiuYH, C. S., Lin CY, Huang Cl, SunYN (2007). Motion tracking on elbow tissue from ultrasonic image sequence for patients with lateral epicondylitis. Conf Proc IEEE Eng Med Biol Soc, 2007, 95 - 98. DO I: 10.1109/ IEM BS.2007.4352231

Lumbroso D, Z. E., Vered E, Kalichman L. (2014). The effect of kinesio tape application on hamstring and gastrocnemius muscles in healthy young adults. J Bodyw Mov Ther, Jan(1). DOI: 10.1016/ j.jbmt.2013.09.011

Maeda N, U. Y., T sutsumi S, Sakai S, Fujishita H, KobayashiT, Asaeda M, Hirda K, MikamiY, Kimura H. (2017). The Acute Effects of Static and Cyclic Stretching on M uscleStiffness and $H$ ardness of M edial Gastrocnemius M uscle.J SportsSci M ed, Dec(16(4)), 514-520.

Moltubakk M M , M. M.,VillarsFO, Seynnes O R, BojsenM øller J. (2018). Specialized properties of thetriceps surae muscle-tendon unit in professional ballet dancers. Scand J Med Sci Sports., Sep(9). DO I: 10.1111/ sms. 13207

OzmenT, A. M., Dogan H, Acar D, ZorogluT, W illems M. (2016). The Effect of Kinesio Taping on M uscle Pain, Sprint Performance, and Flexibility in Recovery From Squat Exercise inYoungAdultWomen. J Sport Rehabil, FEB(1), 7 - 12.

Siqueira CM, R. A., Shimamoto C, Tanaka C. (2018). Balance highly influences flexibility measured by the toe-touch test. Hum M ov Sci, Dec(62), 116-123. DOI: 10.1016/ j.humov.2018.10.001

Stecco C, G. O., Macchi V, Porzionato A, De Caro R, Aldegheri R, DelmasV. (2007). Tendinous muscular insertions onto the deep fascia of the upper limb. First part: anatomical study. Morphologie, Mar(292), 29 - 37. DO I: 10.1016/ j.morpho. 2007.05.001

Rosenthal, R. (1991). Meta analytic procedures for social research (2nd ed.). Newbury Park, CA: Sage.

Turrina A, M.-G. M., Stecco C. (2013). The muscular force transmission system: role of the intramuscular connective tissue. J Bodyw M ov Ther, Jan(1), 95 102. DO I: 10.1016/ j.jbmt.2012.06.001

Witvrouw E, D. L., Asselman P, D'Have T, Cambier. D. (2003). Muscle flexibility as a risk factor for developing muscle injuries in male professional soccer players: a prospective study. Am J Sports Med., 31(1), 41 - 46. doi: 10.1177/ 03635465030310011801 . PMID: 12531755. 